PART III

THEORY OF SPIRAL STRUCTURE 
A. NORMAL SPIRALS 


\title{
58. GRAVITATIONAL THEORIES OF SPIRAL STRUCTURE
}

\author{
G. CONTOPOULOS \\ University of Chicago, Chicago, Ill., U.S.A.*
}

\begin{abstract}
The basic ideas and some of the most important recent developments of the gravitational theories of spiral structure are described. A separation between linear and non linear effects is made. The linear self consistent problem consists of the problem of modes and of the initial value problem, which is discussed here in some detail. More emphasis is put on the non linear problem near resonances and in particular the inner Lindblad resonance. The linear density response to a slightly growing spiral potential (trailing or leading) near the inner Lindblad resonance is always trailing, while non linear effects form a density distribution with a roughly quadruple symmetry.
\end{abstract}

\section{Introduction}

One of the main early difficulties of the gravitational theory of spiral structure was the problem of differential rotation. If the spiral arms were composed always of the same stars then, after a few revolutions, the spiral arms would be wound very tightly and practically disappear. The way out of this difficulty is the notion of spiral arms as waves. Stars move through the spiral arms, but they stay there longer, on the average, so that the spiral arms are, at every moment, the maxima of density; they are not material arms, but spiral waves.

The idea of spiral waves is due to B. Lindblad. In a series of papers, starting in the early forties, he developed many of the elements of the present day theory of spiral waves (Lindblad, 1941, 1942, 1948, 1950; Lindblad and Langebartel, 1953; see also Coutrez, 1947). This is particularly remarkable, in view of the fact that no experience from similar problems in plasma physics was available at that time.

However, although B. Lindblad is the father of the gravitational theory of spiral structure, his views were never widely accepted by astronomers in general. Two reasons, I think, were responsible for that. First the fact that Lindblad's papers are difficult to follow, introducing many assumptions and approximations at every step, and second Lindblad's insistence on leading spirals (except in his last papers before his death). In his paper with Langebartel (1953) it is pointed out that spiral patterns can be both leading or trailing. The authors stress that their "general conclusions ... do not depend on which one of the two alternatives will ultimately prove to be most important". This statement is known to-day to be correct in the general case away from resonances, and, in fact, most of the recent work in spiral wave theory does not discriminate between leading and trailing spirals. However, after making this statement, Lindblad and Langebartel go on discussing in detail only leading spirals.

Thus, the credit goes to C. C. Lin, who not only developed the theory of spiral waves in much more detail, but also presented it in a relatively simple form that made it acceptable to the rest of the astronomical world. The response to the work of Lin

* Present address: University of Thessaloniki, Greece. 
and his associates has been an ever growing wave of research in this area, that has produced many important new results.

In the present Report I will describe some of the main recent developments in this field, including my own recent work.

\section{Outline of the Theory of Spiral Waves}

One can divide the theories of spiral waves into two basic categories: local and global.

Local theories deal with relatively small regions of the galaxy, considering the center to be at distance large with respect to the local dimensions.

A local theory, referring to the gas, was developed by Goldreich and Lynden-Bell (1965) and another one, referring to stars, by Julian and Toomre (1966). Such theories explain the almost universal appearance of small, broken waves in spiral galaxies, which are trailing and may have a rather large inclination angle. Even in quite regular galaxies one sees such 'wavelets' as branches or bridges between the main arms.

However, most of the recent work deals with global theories, which aim at explaining the grand design of the more or less regular two-armed spirals that we see in abundance in the sky. Any irregularities in the spiral pattern are considered, in this approach, as higher order effects, to be introduced, eventually, at a later stage of the theory.

The kinds of problems considered by the theory of spiral structure are given in terms of increasing difficulty, in Table I.

\section{TABLE I}

Theory of spiral waves

\begin{tabular}{ll} 
A. Linear & $\begin{array}{l}\text { 1. Given spiral potential } \\
\text { 2. Self consistent problem } \\
\text { 2a. Modes } \\
\text { 2b. Initial value problem }\end{array}$ \\
\hline B. Non-Linear & $\begin{array}{l}\text { 1. Far from resonances } \\
\text { 2. Near resonances }\end{array}$
\end{tabular}

The first step in developing a theory of spiral waves is the linearization of the collisionless Boltzmann equation

$$
\frac{\partial f}{\partial t}+\mathbf{v} \cdot \frac{\partial f}{\partial \mathbf{x}}-\frac{\partial V}{\partial \mathbf{x}} \cdot \frac{\partial f}{\partial \mathbf{v}}=0
$$

This equation is known also as Vlasov's equation in plasma physics. Here $f$ is the distribution function, while $V$ is the potential of the spiral galaxy. We consider, first, a two-dimensional model of the galaxy; thickness effects can be introduced later.

We assume that $V$ is composed of an unperturbed, axisymmetric, part, $V_{0}(r)$, and 
a spiral part, $V_{1}$, which is small with respect to $V_{0}$. Thus we can write

and

$$
V=V_{0}+V_{1}
$$

$$
f=f_{0}+f_{1}+f_{2}+\cdots,
$$

where $f_{0}$ is the distribution function of the axisymmetric substratum. If we write Equation (1) as

$$
D(f, V)=0
$$

we notice that the operator $D$ is linear in $f$ and in $V$, therefore, if we introduce the value (2) and equate to zero the terms of various orders of Equation (3), we find, first,

$$
D\left(f_{0}, V_{0}\right)=0
$$

this means that $f_{0}$ is a function of the (isolating) integrals of motion of the unperturbed problem. In general, i.e. away from resonances, the only isolating integrals of motion are the angular momentum $J_{0}=r^{2} \oint$ and the energy $E_{o}=\frac{1}{2}\left(\dot{r}^{2}+J_{0}^{2} / r^{2}\right)+V_{0}$, therefore

$$
f_{0}=f_{0}\left(E_{0}, J_{0}\right) \text {. }
$$

The next equation, derived from Equation (3), is

$$
D\left(f_{1}, V_{0}\right)+D\left(f_{0}, V_{1}\right)=0
$$

this is the basic linearized collisionless Boltzmann equation, used extensively in galactic dynamics.

The first problem now consists in finding the response of a galactic disk to a given potential of the form (2).

If the spiral part of the potential, $V_{1}$, is given, the solution of Equation (6) can be written explicitly for every $f_{0}$. In fact Equation (6) is a partial differential equation, with characteristics the unperturbed orbits of the axisymmetric field $V_{0}(r)$, and its solution is

$$
f_{1}=\int P\left(f_{0}, V_{1}\right) \mathrm{d} \tau
$$

where $P$ is an operator linear in $f_{0}$ and $V_{1}$, and the integration is along the unperturbed orbits; $\tau$ is an auxiliary parameter, namely the time along unperturbed orbits, appearing only in trigonometric terms (except in resonances) and after the integration it is expressed in terms of the coordinates.*

In a similar way one can find $f_{2}$, etc.; thus the distribution function $f$ can be found, step by step, as a formal series; $f$ is an integral of motion of the same form as the 'third' integral, found in other galactic problems. In particular if $f_{0}=E_{0}$ we find a 'generalized energy' $E=E_{0}+E_{1}+\cdots$, and if $f_{0}=J_{0}$ we find a 'generalized angular momentum' $J=J_{0}+J_{1}+\cdots$ (Contopoulos, 1967). In the case of a spiral pattern

* This method of solution of the linearized collisionless Boltzmann equation is used extensively in stellar dynamics and plasma physics (see, e.g., Contopoulos, 1960; Shu 1968). 
rotating as a rigid body with angular velocity $\Omega_{\mathrm{s}}$ the Hamiltonian

$$
H=E-\Omega_{\mathrm{s}} J=\frac{1}{2}\left(\dot{r}^{2}+J_{0}^{2} / r^{2}\right)+V-\Omega_{\mathrm{s}} J_{0}
$$

is known to be an analytic integral of motion.

A more difficult problem is the self-consistent (or self-gravitating) problem. In this case $V_{1}$ has to be found, together with $f_{1}$, through Equation (6) and Poisson's equation

$$
\nabla^{2} V_{1}=4 \pi G \delta(z) \sigma_{1}=4 \pi G \delta(z) \int f_{1} \mathrm{~d} \mathbf{v}
$$

where $\delta(z)$ is Dirac's delta function, and $\sigma_{1}$ the perturbed surface density.

Lin and his associates (Lin 1966a, b, 1967a, b; Lin and Shu 1964, 1966, 1967; Lin et al., 1969) have considered in detail the problem of modes. This problem deals with spiral solutions of Equations (7) and (9), of the form

$$
\begin{aligned}
& V_{1}=V_{1}^{*} \exp [i(\omega t-m \vartheta)], \\
& f_{1}=f_{1}^{*} \exp [i(\omega t-m \vartheta)], \\
& \sigma_{1}=\sigma_{1}^{*} \exp [i(\omega t-m \vartheta)],
\end{aligned}
$$

where $V_{1}$ is the spiral component of the potential, $f_{1}$ the corresponding distribution function, and $\sigma_{1}$ the surface density; here $m$ is the number of spiral arms (usually $m=2), \vartheta$ the angle in an inertial frame, and

$$
\omega=m \Omega_{\mathrm{s}} .
$$

The functions $V_{1}^{*}, \sigma_{1}^{*}$ depend only on $r$, while $f_{1}^{*}$ depends on $r$ and the velocities.

If we integrate the solution (7) of Equation (6) over all velocities we find the surface density $\sigma_{1}$. At the same time we replace $V_{1}$ in Equation (7) by a solution of Poisson's Equation (9). Then, if we eliminate the factor $\exp [i(\omega t-m \vartheta)]$, we find an integral equation of the form

$$
r \sigma_{1}^{*}(r)=\int K_{m, \omega}\left(r, r^{\prime}\right) r^{\prime} \sigma_{1}^{*}\left(r^{\prime}\right) \mathrm{d} r^{\prime}
$$

where the kernel $K$ is a complicated function, depending on $m$ and $\omega$. This is the basic integral equation of galactic dynamics. It is of the general form of an homogeneous Fredholm equation of the second kind (the difference is that the dependence on the parameter $\omega$ is not linear, and the equation may be singular). Its eigenvalues $\omega$ are the modes of the self consistent problem and its eigenfunctions $r \sigma_{1}^{*}$ give the corresponding perturbed surface density. This integral equation was given first by Kalnajs (1965) and then in the formalism used in Lin's theory by Shu (1968).

The general solution of this equation is extremely difficult. Thus Lin introduced an 'asymptotic' approximation that simplifies the problem considerably and makes it tractable. The 'asymptotic' approximation consists in assuming the radial wavelength $\lambda$ of the spiral pattern as small and omitting all higher order terms in $\lambda$. Then the integral equation is reduced to an algebraic relation between $\omega$, the wave number 
$k=2 \pi / \lambda$, and $r$,

$$
D(\omega, k, r) \equiv 1-\frac{2 \pi G \sigma_{0}}{|k|\left\langle\dot{r}^{2}\right\rangle}\left[1-\frac{v \pi}{\sin v \pi} \boldsymbol{G}_{v}\left(\chi_{*}\right)\right]=0 .
$$

Here $\sigma_{0}$ is the surface density of the basic, axisymmetric, distribution, $\left\langle\dot{r}^{2}\right\rangle^{\frac{1}{2}}$ the velocity dispersion, $v$ is the 'relative frequency' defined by

$$
v=(\omega-2 \Omega) / \kappa,
$$

with $\Omega$ the angular velocity of the galactic rotation and $\kappa$ the 'epicyclic frequency' at distance $r$,

and

$$
\chi_{*}=k^{2}\left\langle\dot{r}^{2}\right\rangle / \kappa^{2}
$$

$$
\mathfrak{G}_{v}\left(\chi_{*}\right)=\frac{1}{2 \pi} \int_{-\pi}^{\pi} \cos v \gamma \exp \left[-\chi_{*}(1+\cos \gamma)\right] \mathrm{d} \gamma .
$$

The relation (13) is Lin's dispersion relation (Lin, 1966a) for a Schwarzschild distribution of unperturbed velocities.

By solving this equation for a given $\omega$ we find $k=k_{\omega}(r)$. Then the spiral arms, in a frame of reference rotating with the spiral field, are given by

$$
\vartheta^{\prime}=\frac{1}{2} \int k_{\omega}(r) \mathrm{d} r+\operatorname{const}(+\pi),
$$

where

$$
\vartheta^{\prime}=\vartheta-\Omega_{\mathrm{s}} t .
$$

Lin found which spirals of the form (17) fit best the spiral arms of our Galaxy and in this way he derived a value of $\Omega_{\mathrm{s}}=\omega / 2$ near $13 \mathrm{~km} \mathrm{~s}^{-1} \mathrm{kpc}^{-1}$.

A confirmation of this value of the angular velocity of the spiral pattern, $\Omega_{\mathrm{s}}$, came from a rather different approach by Fujimoto at Columbia University. Fujimoto (1968) studied in particular gaseous spiral arms, using the hydrodynamic equations instead of the collisionless Boltzmann Equation. He solved the linearized equations numerically and found the density response to various imposed spiral potentials. Imposing self-consistency, i.e. agreement between the phase and amplitude of the response with the density responsible for the spiral field he could find a value of $\Omega_{\mathrm{s}}$ similar to that of Lin, and, further, a relation between the inclination of spiral arms and the proportion of gas in them; more open spirals contain more gas.

Accurately speaking a linear theory cannot give the absolute value of the amplitude of the wave, because if $\sigma_{1}^{*}$ is a solution of Equation (12), or of a similar linear equation, so is also $c \sigma_{1}^{*}$, where $c$ is an arbitrary constant. Thus Fujimoto considered only the relative variations of the response with the radius $r$. The absolute value of the amplitude can be found only by a non-linear theory. This has been done recently by Vandervoort and will be reported during this Symposium.

Kalnajs has recently solved numerically the integral Equation (12) and has found values of $\Omega_{\mathrm{s}}$ of the order of $30 \mathrm{~km} \mathrm{~s}^{-1} \mathrm{kpc}^{-1}$. His spirals are rather open and cannot 
be treated by an 'asymptotic' theory, like Lin's. Most of Kalnajs' original work is contained in his Thesis (1965), while his recent work will be reported during the present Symposium. The problem of open spirals is quite difficult and more effort should be turned in this direction.

Let us now turn to the initial value problem. This is the problem of the evolution of a given initial perturbation (given at time $t=0$ ).

This problem is well known in plasma physics. In the case of an homogeneous plasma its solution by Landau (1946) is by now classical. However in the galactic case only Kalnajs (1965) mentioned it briefly.

One can solve, in principle, the initial value problem after the problem of modes has been solved. The solution is found by a variation of Landau's method of Fourier transforms in space and a Laplace transform in time. Namely we perform a Fourier analysis in the angle $\vartheta$, followed by a Laplace transform in time, omitting a Fourier transform in $r$.

It is obvious that any perturbation of an axisymmetric galaxy, being a periodic function in the angle $\vartheta$, with period $2 \pi$, can be Fourier analyzed into a one-armed perturbation, a two-armed perturbation, etc. Let us consider only two-armed perturbations. Then we can write

$$
\begin{aligned}
& V_{1}=V_{11} \exp (-2 i \vartheta), \\
& f_{1}=f_{11} \exp (-2 i \vartheta), \\
& \sigma_{1}=\sigma_{11} \exp (-2 i \vartheta) .
\end{aligned}
$$

The initial perturbation, at $t=0$, is also written

$$
f_{1 ; 0}=f_{1 ; 0}^{*} \exp (-2 i \vartheta) \text {, }
$$

where $f_{1 ; 0}^{*}$ is a function of $r$ and the velocities. We can write

$$
f_{1 ; 0}^{*}=f_{0} a \exp (i \phi),
$$

where $f_{0}$ is the unperturbed distribution function, $a$ a relative amplitude and $\phi$ a phase angle.

Then we take the Laplace transforms of $V_{11}, f_{11}$ and $\sigma_{11}$

$$
f_{1}^{*}=\int_{0}^{\infty} f_{11} \exp (-i \omega t) \mathrm{d} t
$$

and similar expressions for $V_{1}^{*}$ and $\sigma_{1}^{*}$. Thus Equation (6) gives a differential equation for $f_{1}^{*}$

$$
i\left(\omega-\frac{J_{0}}{r^{2}}\right) f_{1}^{*}+\dot{r} \frac{\mathrm{d} f_{1}^{*}}{\mathrm{~d} r}=\frac{\partial f_{0}}{\partial E_{0}} \dot{r} \frac{\mathrm{d} V_{1}^{*}}{\mathrm{~d} r}-2 i V_{1}^{*}\left(\frac{\partial f_{0}}{\partial E_{0}} \frac{J_{0}}{r^{2}}+\frac{\partial f_{0}}{\partial J_{0}}\right)+f_{1 ; 0}^{*},
$$

under the restriction

$$
\lim _{t \rightarrow \infty}\left[f_{11} \exp (-i \omega t)\right]=0 .
$$


Equation (23) is the same as the equation for the modes (Shu, 1968) except for the last term $f_{1 ; 0}^{*}$. If we solve it for $f_{1}^{*}$ and integrate over all velocities we find an integral equation very similar to Equation (12), namely

$$
r \sigma_{1}^{*}(r)=\int K_{m, \omega}\left(r, r^{\prime}\right) r^{\prime} \sigma_{1}^{*}\left(r^{\prime}\right) \mathrm{d} r^{\prime}+s_{10}(r),
$$

Where $s_{10}(r)$ is a known function, depending on the initial conditions. This is of the general form of a nonhomogeneous Fredholm equation of the second kind, and its solution can be given once the solution of the homogeneous Equation (12) is known.

In the asymptotic case the solution can be given by using the formalism of Lin and his associates. In the Appendix we derive the solution for the perturbed density $\sigma_{1}$ in the form

$$
\sigma_{1}=\sigma_{0} a \exp [i(\phi-\pi / 2-2 \vartheta)] \frac{1}{2 \pi \kappa} \int_{-\infty+i \omega_{\mathbf{I}}}^{\infty+i \omega_{\mathbf{I}}} \frac{1}{\sin v \pi} \frac{\mathfrak{F}_{\nu}\left(\chi_{* ; 0}\right)}{D} \exp (i \omega t) \mathrm{d} \omega
$$

where $D$ is the function (13) and $i \omega_{\mathrm{I}}$ is the imaginary part of $\omega=\omega_{R}+i \omega_{\mathrm{I}}$ along the line of integration; this line is drawn below all singularities of the integrand, which are assumed to be poles.

Following Landau we move the line of integration in the integral (26) parallel to itself, so that it comes at $i \omega^{\prime}$ with $\omega^{\prime}>0$. Then it is known that for large $t$ the only contribution to $\sigma_{1}$ comes from the poles of the integrand. If we are not at a resonance (i.e. when $\sin v \pi \neq 0$ ) the only poles are the roots of the equation $D=0$, i.e. Lin's modes. Thus the problem of the origin of spiral waves becomes, in some sense, trivial. Because practically any two-armed initial perturbation excites Lin's modes. (Of course the appearance of other modes also is not excluded, especially in open spirals.)

However, there is a difference between this result and Lin's original picture of the modes. The value of $\omega$, which is a solution of Equation (13), is not unique. In fact the solution $\sigma_{1}$ (Equation (26)), gives the wave number $k$ as a function of $r$ and $t$,

$$
k=k(r, t),
$$

thus Equation (13) gives also $\omega$ as a function of $r$ and $t$,

$$
\omega=\omega(r, t) \text {. }
$$

Relations of this form have been used as the starting point of Toomre's recent work (1969) on the evolution of the density waves in a galaxy. The fact that $\omega$ is not constant along a spiral wave produces a differential rotation, affecting the wave itself, which tends to produce the ultimate dissolution of spiral waves.

Toomre finds that the 'group velocity' of the spiral waves, $\mathrm{d} \omega / \mathrm{d} k$, is directed inwards in the main part of the galaxy and of the order of $10 \mathrm{~km} \mathrm{~s}^{-1}$. Any information contained in the spiral waves moves inwards with this group velocity. Toomre found that certain quantities, like the wave number and the action density (energy density 
divided by the relative frequency $v$ ) are preserved, as they are transmitted with the group velocity. Thus the whole wave pattern moves towards the inner Lindblad resonance. Toomre suspects that the energy of the wave is transformed there to thermal energy of the stars, and the wave is damped. In a numerical example he found that the amplitude of the wave decreases as the wave moves inwards, and tends to zero near the inner Lindblad resonance. This damping of the wave happens in about $10^{9}$ years, therefore a mechanism is needed to regenerate the spiral waves. Various suggestions of 'exterior' forcing mechanisms were proposed by Toomre, including the Magellanic Clouds or a small bar near the center of the Galaxy.

However Toomre's conclusion is not generally accepted. An alternative assumption, invoking a reflection of the wave near the inner Lindblad resonance, will be presented by Lin during this Symposium. At any rate near the inner Lindblad resonance strong non-linear phenomena take place and as we will see presently, any linear theory is not applicable there except for short times.

\section{Non-Linear Theory}

Away form resonances non-linear effects appear only as small corrections in $f_{1}$ (higher order terms $f_{2}$, etc.). Their main function is in stopping the growth of a finite, growing, wave, so that it reaches a stationary state. Then one can calculate the amplitude reached by the wave (Vandervoort).

Non-linear effects are extremely important near resonances. There the whole linear theory is inapplicable for long times. In fact in the response integral (7), as given by Lin and his associates, there is a denominator $\sin v \pi$, and this becomes very small if $v$ is near an integer. Then $f_{1}$ is larger than $f_{0}$ and the whole approximation scheme implied by the linearization is not valid. In particular, if $v$ is exactly an integer, $f_{1}$ contains a secular term; therefore the linear theory can be applied only for a short time.

Whenever $v$ is an integer (or a rational number) we have a resonance between the frequency of rotation in a frame of reference rotating with the spiral pattern, and the epicyclic frequency; the unperturbed orbits in the rotating frame are closed, periodic, orbits. The most important resonance in a galaxy is the inner Lindblad resonance, where

$$
v=-1
$$

In order to solve the collisionless Boltzmann equation in this case we cannot start with $f_{0}$ a function of the energy $E_{0}$ and the angular momentum $J_{0}$, because then $f_{0}$ contains secular terms, except in the quite special case that $f_{0}$ is a function of the Hamiltonian only.

However, in the case $v=-1$ we have one more isolating integral of the axisymmetric problem, namely the initial phase difference between the motion around the center of the galaxy and the radial (epicyclic) motion. We use this integral in the form

$$
\begin{aligned}
& S_{0} \\
& C_{0}
\end{aligned}=\frac{\sin }{\cos }\left(2 \vartheta_{1}-\omega \tau_{1}\right),
$$


where $\vartheta_{1}$ and $\tau_{1}$ are the angle and the corresponding time of a pericentron passage.

We can now find a function $f_{0}$ of $E_{0}, J_{0}$ and $S_{0}$ (or $C_{0}$ ), such that it does not produce secular terms in $f_{1}$. This means that $f_{0}$ cannot be a Schwarzschild distribution function, but has a more complicated form. The main result (Contopoulos, 1970a) is that $f_{0}$ has a $4 \vartheta^{\prime}$ dependence, where $\vartheta^{\prime}$ is the angle (17) in the rotating frame. Namely the main term of $f_{0}$ contains $\cos \left[4 \vartheta^{\prime}+\Phi_{1}(r)\right]$, where $\Phi_{1}$ is a phase function.

This quadruple symmetry of $f_{0}$ is evident in the form of the orbits near the inner Lindblad resonance. Away from resonances the orbits fill rings around the center (Figure 1); for every given value of the Hamiltonian (8) there is one almost circular

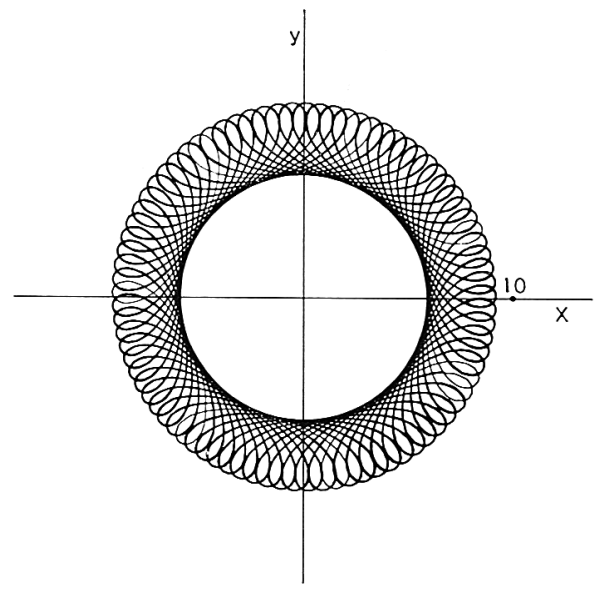

Fig. 1. A ring-type orbit.

periodic orbit, and all the rest form rings with boundaries on each side of the periodic orbit. Near the inner Lindblad resonance, however, for every value of the Hamiltonian there are two resonant periodic orbits, like ellipses with their center at the center of the Galaxy. Orbits near the resonance (e.g. starting at a distance up to $2 \mathrm{kpc}$ from the resonance, with appropriate values of the Hamiltonian) form tube orbits around the two resonant periodic orbits (Figure 2). Orbits starting further away from the resonance form rings, as in Figure 1, but of larger width; the set of such orbits, however, is small.*

* The other resonances, besides the inner Lindblad resonance, are less important, because they involve much smaller sets of orbits. A particularly interesting resonance is the particle resonance, where $v=0$, which will be discussed by Barbanis.

Resonances are encountered also in many problems of stellar dynamics and celestial mechanics. Tube orbits in a meridian plane of an axisymmetric galaxy were found by Torgard and Ollongren (1960) (see also Ollongren, 1965). Their theoretical explanation, as resonance phenomena, was given by means of the 'third' integral (Contopoulos, 1965) and follows the lines discussed above. Excellent agreement was found between theory and numerical experiments, by using an extra integral, like $S_{0}$ (or $C_{0}$ ), in the resonant case. The analogy with the present problem is discussed in more detail in a forthcoming paper (Contopoulos, 1970a).

In celestial mechanics the same problem is known as the problem of 'small divisors'. Resonances appear in the gaps of the asteroids, in satellite orbits at the 'critical inclination', etc. 
The superposition of all the orbits near the inner Lindblad resonance gives the density response. This has also a rough quadruple symmetry. In fact, in some of the galaxies shown by Morgan during this Symposium, one could see nuclei showing a rough quadruple symmetry.

It is obvious that if the spiral field is infinitesimal resonant effects are also infinitesimal. In order to see the growth of a resonance we have calculated numerically orbits in a growing wave (Contopoulos, 1970a). Initially the spiral potential $V_{1}$ is zero and the stars start moving along their unperturbed (epicyclic) orbits. However as the wave gradually grows, tending to its maximum amplitude, the orbits deviate gradually,

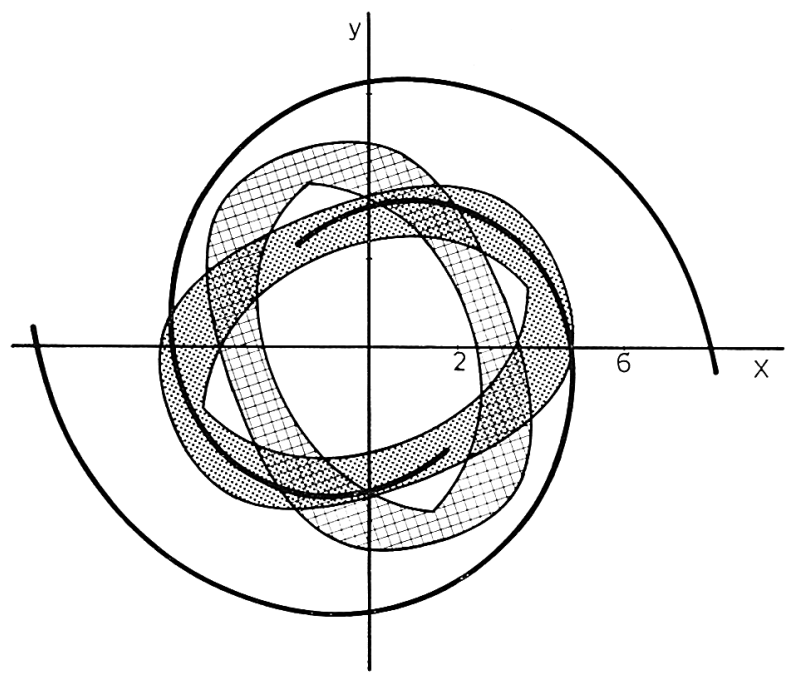

Fig. 2. The areas covered by two tube orbits near the inner Lindblad resonance. The thick line gives the minimum of the spiral potential.

tending to their final tube form around one or the other of the two resonant periodic orbits. In a particular example the field reaches 0.9 of its maximum amplitude in about $2 \times 10^{9}$ years, while the orbits reach almost exactly their final tube forms after at most 10 revolutions. Therefore the growth of resonances is an important factor in the evolution of a spiral wave.

We must stress that any 'absorption' or 'reflection' of the waves near the inner Lindblad resonance is different from the corresponding linear phenomena, known from plasma physics, which happen away from resonances. Lin will mention the possibility of the excitation of a long outgoing wave near the inner Lindblad resonance which may be considered as a non-linear reflection. If such a wave exists it may also have important consequences for the dynamics of spiral waves.

\section{Preference of Trailing Waves}

It is generally believed to-day that spiral waves are trailing rather than leading. This 
is well known for local, sheared, wavelets (Goldreich and Lynden-Bell, 1965; Julian and Toomre, 1966), but in the case of the grand design of spiral waves leading and trailing waves appear as equivalent. Some 'indications' for the amplification of trailing waves (Lin and Shu 1966; Lin 1967a) have not proved working (Toomre, 1969). The only evidence for the preference of trailing waves has been provided by Kalnajs (1965), who states that the response to a trailing wave is trailing, while the response to a leading wave is both a leading and a trailing wave. Kalnajs' argument is correct, but difficult to follow, and applies to the general case of open spirals.

Thus we made some calculations following Lin and Shu's formalism, for a slightly growing wave near the inner Lindblad resonance. Namely we calculated the linear response, near the inner Lindblad resonance, of a spiral wave of the form

$$
V_{1}=A(r) \exp \{i[\Phi(r)+\omega t-2 \vartheta]\},
$$

where $\omega=\omega_{R}+i \omega_{1}$ has a small negative imaginary part, which gives a slightly growing wave (because $V_{1}$ contains the factor $\exp \left(-\omega_{1} t\right)$ ). The value of $\left|\omega_{1}\right|$ was taken equal to $0.03 \omega_{R}$. The results of the calculation are shown in Figure 3. It is seen that near resonance the response to a trailing wave is trailing, while the response to a leading wave is also trailing.
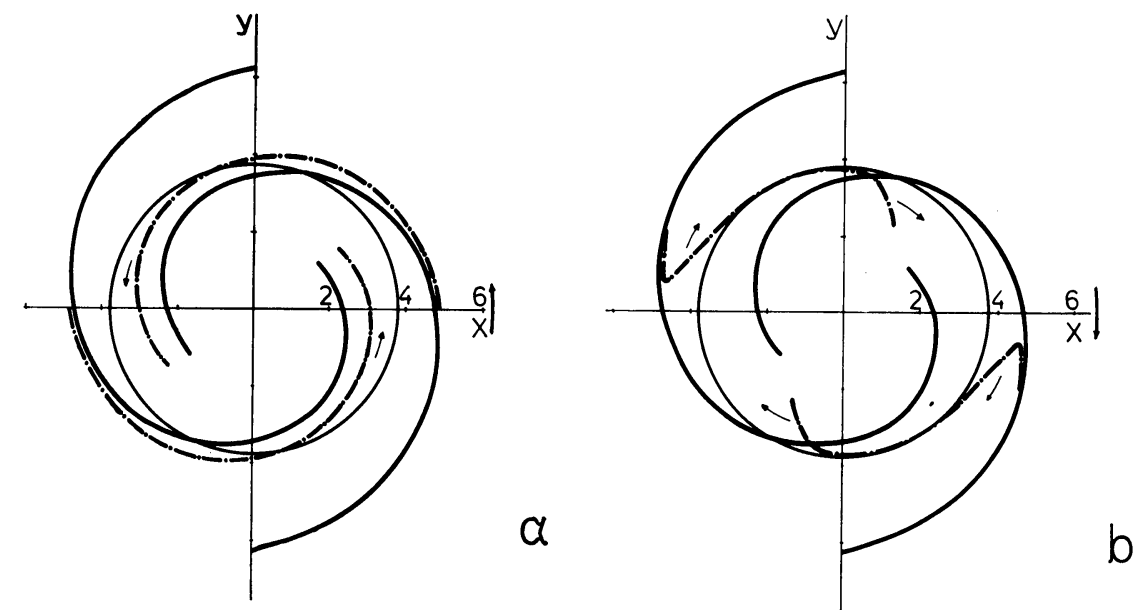

Fig. 3. Linear density response to a growing imposed field near the inner Lindblad resonance (thin circle). The thick solid line represents the minimum of the potential, which is trailing in (a) and leading in (b). The response (dash-dotted line) is trailing in both cases.

It is known from Lin's theory that outside the inner Lindblad resonance the response is almost exactly in phase with the imposed potential, i.e. the maxima of density almost coincide with the minima of potential. Inside the Lindblad resonance the response is $90^{\circ}$ out of phase. Therefore there cannot be a self consistent spiral wave inside the inner Lindblad resonance, at least in the linear theory. This can be well understood in terms of the initial value problem. As the Equation (13) has no solution inside the inner Lindblad resonance, the integrand of Equation (26) has no poles, therefore the solution (26) does not tend to a stationary spiral form after a long time. 
This is consistent with observations of the nuclear region of galaxies and with the numerical experiments of Miller and Prendergast.

In the case of an imposed growing spiral field it is not at all evident how the in-phase response outside the inner Lindblad resonance is joined to the out-of-phase response inside it. However an exact linear calculation shows that in both cases of a trailing and of a leading wave the response precedes the imposed potential, and more strongly so in the case of the leading wave (Figure 3). Thus a leading wave is strongly distorted near the inner Lindblad resonance tending to become trailing. This effect discriminates strongly in favor of trailing waves. More details will be given in a future paper (Contopoulos, 1970b). It appears that permanent leading waves are impossible in galaxies possessing an inner Lindblad resonance.

The above review of the recent developments in the theory of spiral structure shows that much progress has been made in recent years. However there are still some basic unsolved problems. Perhaps the most urgent at this moment is to find what is the fate of the groups of waves moving towards the inner Lindblad resonance with Toomre's group velocity. The complete exploration of the non-linear effects near resonances may give the answer to the basic problems of the origin and persistence of spiral waves.

\section{Appendix. The Initial Value Problem in the Asymptotic Approximation}

The solution of Equation (23) of the initial value problem can be found in the same way as the solution of the corresponding equation for modes (Shu, 1968). It is

$$
\begin{aligned}
f_{1}^{*}= & \frac{\partial f_{0}}{\partial E_{0}} V_{1}^{*}-\frac{1}{2 \sin \left(\omega \tau_{0}-2 \vartheta_{0}\right)} \\
& \times \int_{-\tau_{0}}^{\tau_{0}}\left\{V_{1}^{*}\left(2 \frac{\partial f_{0}}{\partial J_{0}}+\omega \frac{\partial f_{0}}{\partial E_{0}}\right)+i f_{1 ; 0}^{*}\right\} \cos [\omega \tau-2 \vartheta(\tau)] \mathrm{d} \tau .
\end{aligned}
$$

Then, using a Schwarzschild distribution function

$$
f_{0}=\frac{\Omega_{0} \sigma_{0}\left(r_{0}\right)}{\pi \kappa_{0}\left\langle\dot{r}^{2}\right\rangle_{0}} \exp \left\{-\frac{\left[\dot{r}^{2}+\kappa_{0}^{2}\left(r_{0}-r\right)^{2}\right]}{2\left\langle\dot{r}^{2}\right\rangle_{0}}\right\},
$$

(where a zero subscript in $\Omega, \kappa,\left\langle\dot{r}^{2}\right\rangle$ means quantities calculated at $r=r_{0}$ ) and the approximate relation (Shu, 1968)

$$
2 \frac{\partial f_{0}}{\partial J_{0}}+\omega \frac{\partial f_{0}}{\partial E_{0}}=-\frac{f_{0} v_{0} \kappa_{0}}{\left\langle\dot{r}^{2}\right\rangle_{0}}
$$

we find, using the same approximations as Shu,

$$
f_{1}^{*}=\frac{f_{0}}{\left\langle\dot{r}^{2}\right\rangle_{0}}\left\{-V_{1}^{*}+\frac{v_{0}}{2 \sin v_{0} \pi} \int_{-\pi}^{\pi}\left[V_{1}^{*}-\frac{i\left\langle\dot{r}^{2}\right\rangle_{0}}{v_{0} \kappa_{0}} a \exp (i \phi)\right] \cos v_{0} \gamma \mathrm{d} \gamma\right\} \text {. }
$$


Integrating over all velocities we find the density response. If we assume that $a$ and $\phi$ depend only on $r$ and not on the velocities, we find

$$
\sigma_{1}^{*}=\frac{-\sigma_{0} V_{1}^{*}}{\left\langle\dot{r}^{2}\right\rangle}\left\{1-\frac{v \pi}{\sin v \pi}\left(_{v}\left(\chi_{*}\right)\right\}-\frac{i \pi \sigma_{0} a \exp (i \phi)}{\kappa \sin v \pi} \mathfrak{G}_{v}\left(\chi_{* ; 0}\right),\right.
$$

where $\mathfrak{F}_{v}\left(\chi_{*}\right)$ is given by Equation (16), and $\chi_{* ; 0}=\left(\phi^{\prime}\right)^{2}\left\langle\dot{r}^{2}\right\rangle / \kappa^{2}$, where $\phi^{\prime}=k$ at $t=0$. If the last assumption about $a$ and $\phi$ is not made the last term of Equation (A5) is slightly more complicated.

If we replace $V_{1}^{*}$ by the lowest order solution of Poisson's equation, $V_{1}^{*}=-2 \pi \times$ $G \sigma_{1}^{*} /|k|$, and omit the second term of Equation (A5) we find Lin's dispersion relation (Equation (13)).

In the initial value problem $D$ is not zero, in general, because of the second member in (A5). The solution of Equation (A5) is

$$
\sigma_{1}^{*}=\frac{\pi \sigma_{0} a \exp [i(\phi-\pi / 2)]}{\kappa \sin v \pi} \frac{\left(\mathfrak{G}_{v}\left(\chi_{* ; 0}\right)\right.}{D} .
$$

Then, inverting the Laplace transform (22), and using Equation (19), we find the solution of the initial value problem in the form (26).

If we move the line of integration above the real axis of $\omega$, then, for large $t$, only the poles of the integrand give a contribution to $\sigma_{1}$.

Let us consider the main root of the dispersion relation $D=0$, say $\omega=\omega(r, k)$. Then we find

$$
\sigma_{1}=\frac{\sigma_{0} a \exp [i(\phi-2 \vartheta)]}{\sin v \pi} \frac{\left(\mathfrak{G}_{v}\left(\chi_{* ; 0}\right)\right.}{\partial D / \partial v} \exp (i \omega t)
$$

where it is assumed that $\sin v \pi \neq 0$.

Equation (A7) gives the form of the perturbed density distribution for every (large) $t$, therefore it gives also the wave number $k$. We know that initially (for $t=0$ ) it is

$$
k(r)=\phi^{\prime}(r) .
$$

Let us disregard the transition period needed for $\sigma_{1}$ to reach the form (A7) and consider a new 'initial time', such that (A7) is satisfied approximately for every $t \geqslant 0$. Then we have two possibilities.

(a) If the initial perturbation satisfies Lin's dispersion relation for a fixed $\omega$, i.e. if $k(r)$, defined by Equation (A8), satisfies the equation $D(\omega, k(r), r)=0$, then this mode is stationary, except for resonance effects.

(b) If the initial perturbation does not satisfy the above condition, then, for $t=0$, $\omega$ is not constant, but a function of $r$. Equation (A7) gives at time $\Delta t$

$$
k(r)=\phi^{\prime}(r)+\omega^{\prime}(r) \Delta t
$$

therefore the wave number changes in time. Then the dispersion relation gives $\omega$ as a function of $r$ and $t$, i.e. the spiral pattern has a differential rotation. 


\section{References}

Contopoulos, G.: 1960, Z. Astrophys. 49, 273.

Contopoulos, G.: 1965, Astron. J. 70, 526.

Contopoulos, G.: 1967, Proceedings 14th Liege Colloquium, p. 213.

Contopoulos, G.: 1970a, Astrophys. J., in press.

Contopoulos, G.: 1970b, Astrophys. J., to be published.

Coutrez, R.: 1947, Stockholm Obs. Ann. 15, No. 3.

Fujimoto, M.: 1968, Astrophys. J. 152, 391.

Goldreich, P. and Lynden-Bell, D.: 1965, Monthly Notices Roy. Astron. Soc. 130, 125.

Julian, W. H. and Toomre, A.: 1966, Astrophys. J. 146, 810.

Kalnajs, A.: 1965, Ph.D. Thesis, Harvard University.

Landau, L.: 1946, J.Phys. U.S.S.R. 10, 25.

Lin, C. C.: 1966a, SIAM J. Appl. Math. 14, 876.

Lin, C. C.: 1966b, in Galaxies and the Universe, Columbia University Press. New York and London, p. 33.

Lin, C. C.: 1967a, in Relativity Theory and Astrophysics, 2: Galactic Structure, Amer. Math. Soc., Providence, p. 66.

Lin, C. C.: 1967b, Ann. Rev. Astron. Astrophys. 5, 453.

Lin, C. C. and Shu, F. H.: 1964, Astrophys. J. 140, 646.

Lin, C. C. and Shu, F. H.: 1966, Proc. Nat. Acad. Sci. U.S.A. 55, 229.

Lin, C. C. and Shu, F. H.: 1967, IAU-URSI Symposium No. 31, p. 313.

Lin, C. C., Yuan, C., and Shu, F. H.: 1969 Astrophys. J. 155, 721.

Lindblad, B.: 1941, Stockholm Obs. Ann. 13, No. 10.

Lindblad, B.: 1942, Stockholm Obs. Ann. 14, No. 1.

Lindblad, B.: 1948, Stockholm Obs. Ann. 15, No. 4.

Lindblad, B.: 1950, Stockholm Obs. Ann. 16, No. 1.

Lindblad, B. and Langebartel, R.: 1953, Stockholm Obs. Ann. 17, No. 6.

Ollongren, A.: 1965, Ann. Rev. Astron. Astrophys. 3, 113.

Shu, F. H.: 1968, Ph.D. Thesis, Harvard University.

Toomre, A.: 1969, Astrophys. J. 158, 899.

Torgard, I. and Ollongren A.: 1960, NUFFIC Intern. Summer Course in Science, Part X. 